Research Article

\title{
Analysis of $\mathrm{SC}_{5} \mathrm{C}_{7}[p, q]$ and $\mathrm{NPHX}[p, q]$ Nanotubes via Topological Indices
}

\author{
Ye-Jun Ge, ${ }^{1}$ Jia-Bao Liu $\left(\mathbb{D},{ }^{2}\right.$ Muhammad Younas, ${ }^{3}$ Muhammad Yousaf, ${ }^{3}$ \\ and Waqas Nazeer iD ${ }^{4}$ \\ ${ }^{1}$ School of Materials and Chemical Engineering, Anhui Jianzhu University, Hefei 230601, China \\ ${ }^{2}$ School of Mathematics and Physics, Anhui Jianzhu University, Hefei 230601, China \\ ${ }^{3}$ Department of Mathematics, COMSATS University Islamabad, Lahore Campus, Lahore 54000, Pakistan \\ ${ }^{4}$ Division of Science and Technology, University of Education, Lahore 54000, Pakistan
}

Correspondence should be addressed to Waqas Nazeer; nazeer.waqas@ue.edu.pk

Received 7 November 2018; Accepted 24 February 2019; Published 23 April 2019

Academic Editor: Stefano Bellucci

Copyright (c) 2019 Ye-Jun Ge et al. This is an open access article distributed under the Creative Commons Attribution License, which permits unrestricted use, distribution, and reproduction in any medium, provided the original work is properly cited.

Scientists are creating materials, for example, a carbon nanotube-based composite created by NASA that bends when a voltage is connected. Applications incorporate the use of an electrical voltage to change the shape (transform) of air ship wings and different structures. Topological indices are numbers related with molecular graphs to allow quantitative structure activity/property/poisonous relationships. Topological indices catch symmetry of molecular structures and give it a scientific dialect to foresee properties, for example, boiling points, viscosity, and the radius of gyrations. We compute $M$-polynomials of two nanotubes, $\mathrm{SC}_{5} \mathrm{C}_{7}[p, q]$ and $\mathrm{NPHX}[p, q]$. The closed form of $M$-polynomials for these nanotubes produces formulas of numerous degree-based topological indices which are functions relying on parameters of the structure and, in combination, decide properties of the concerned nanotubes. Moreover, we sketch our results by using Maple 2015 to see the dependence of our results on the involved parameters.

\section{Introduction}

The expression "nano" alludes to the metric prefix $10^{-9}$. It implies one billionth of something. "Nano" can be attributed to any unit of measure. For instance, you may report a little mass in nanograms or the measure of fluid in one cell as far as nanoliters. Nanoscience is the investigation of structures and materials on the size of nanometers. When structures are made enough in the nanometer measure range, they can go up against fascinating and valuable properties. Nanoscale structures have existed in nature well before researchers started considering them in labs. Researchers have even made nanostructures in the lab that copy a portion of nature's stunning nanostructures [1-3].

Nanoscience has effectively affected our lives with developments, for example, stain-resistant fabrics propelled by nanoscale highlights found on lotus plants and PC hard drives, which store data on magnetic strips that are only 20 nanometers thick. Researchers from several disciplines material science, biology, chemistry, and physics utilize nanoscience standards for applications in computing, medication, energy, information storage, etc. In spite of the fact that achievements in any research field are hard to anticipate, the eventual fate of nanoscience will probably include scaling up from atomic get together and singular nanodevices to macroscopic systems and structures with developing properties and different capacities. Carbon nanotubes can be utilized as the pores in layers to run reverse osmosis desalination plants. Water atoms go through the smoother walls of carbon nanotubes more effortlessly than through different sorts of nanopores, which requires less power. Different scientists are utilizing carbon nanotubes to develop little, inexpensive water purification devices required in poor nations. Sensors utilizing carbon nanotube detection elements are fit 


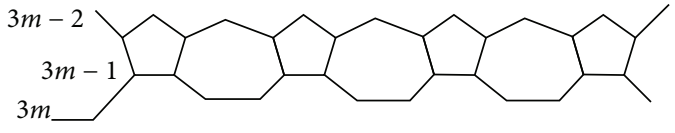

FIgURE 1: $i$ th period of $\mathrm{SC}_{5} \mathrm{C}_{7}[p, q]$ nanotube.

for distinguishing a scope of chemical vapors. These sensors work by responding to the changes in the resistance of a carbon nanotube within the sight of a chemical vapor [4-7].

The features of energetics and electronic properties of carbon nanotubes, containing a pentagon-heptagon pair $(5 / 7)$ topological defect in the hexagonal network of the zigzag configuration, are investigated using the extended SuSchriffer-Heeger model based on the tight binding approximation in real space. Calculations show that this pentagonheptagon pair defect in the nanotube structures is not only responsible for a change in nanotube diameter but also governs the electronic behavior around the Fermi level [8].

In nanoscience, $\mathrm{SC}_{5} \mathrm{C}_{7}[p, q]$ (where $p$ and $q$ express the number of heptagons in each row and the number of periods in whole lattice, respectively) nanotube is a class of $\mathrm{C}_{5} \mathrm{C}_{7}$ net which is yielded by alternating $\mathrm{C}_{5}$ and $\mathrm{C}_{7}$. The standard tiling of $\mathrm{C}_{5}$ and $\mathrm{C}_{7}$ can cover either a cylinder or a torus, and each period of $\mathrm{SC}_{5} \mathrm{C}_{7}[p, q]$ consisted of three rows (more details on $p$ th period can be referred to in Figure 1). H-Naphtalenic nanotubes $\operatorname{NPHX}[p, q]$ (where $p$ and $q$ are denoted as the number of pairs of hexagons in first row and the number of alternative hexagons in a column, respectively) are a trivalent decoration with sequence of $\mathrm{C}_{6}, \mathrm{C}_{6}, \mathrm{C}_{4}, \mathrm{C}_{6}, \mathrm{C}_{6}, \mathrm{C}_{4}, \ldots$ in the first row and a sequence of $\mathrm{C}_{6}, \mathrm{C}_{8}, \mathrm{C}_{6}, \mathrm{C}_{8}, \ldots$ in the other rows. In other words, this nanolattice can be considered as a plane tiling of $\mathrm{C}_{4}, \mathrm{C}_{6}$, and $\mathrm{C}_{8}$. Therefore, this class of tiling can cover either a cylinder or a torus [9].

In the field of chemical graph theory, the molecular graph $G$ is a simple connected graph in which atoms are taken as vertices and chemical bonds are taken as edges. The vertex set is usually denoted by $V$, and the edge set is denoted by $E$. The degree of a vertex $v$ is denoted by $d_{v}$, which is the total number of vertices attached with it. The valence in chemistry and the degree of a vertex in a graph are closely related. For details about graph theory and its notion, we refer to the book [10].

Another emerging field is cheminformatics. In cheminformatics, the quantitative structure-activity (QSAR) and the structure-property (QSPR) relationship foresees the biological activity and properties of understudy nanomaterial. In these investigations, topological indices and some physicochemical properties are utilized to foresee bioactivity of understudy nanomaterial, see [11-15]. Polynomials have additionally valuable applications in chemical graph theory, for example, Hosoya polynomial (also called Wiener polynomial) [16] which assumes an indispensable job in deciding distance-based topological indices. Among other polynomials, $M$-polynomial [17] presented in 2015 assumes a similar job in deciding the closed form of many degree-based topological indices [18-22]. The primary favorable position of $M$-polynomial is the abundance of data that it contains about degree-based graph invariants.

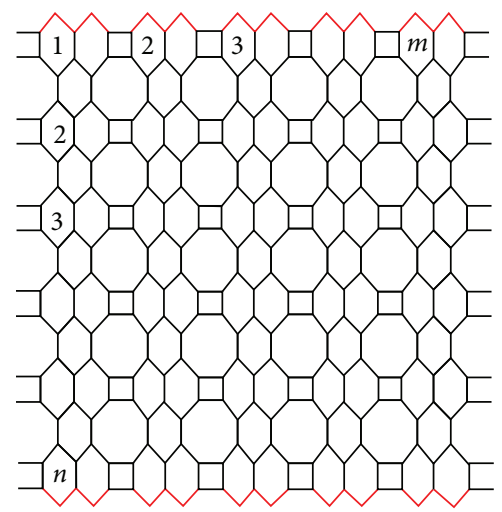

Figure 2: Naphtalenic nanotubes.

In this paper, we focus on the degree-based combinatorial facts of two nanotubes. At first, we give $M$-polynomial of these nanotubes shown in Figures 1 and 2. Then, by using calculus rules on the $M$-polynomials, we recover nine topological indices.

\section{Basic Definition and Literature Review}

In this section, we give basic definitions and notions. Throughout this paper, we fix the following notion.

(1) $G=$ connected simple graph

(2) $V(G)=$ set of vertices of $G$

(3) $E(G)=$ set of edges

(4) $|V(G)|=$ numbers of elements in $V(G)$

(5) $|E(G)|=$ numbers of elements in $E(G)$

(6) $d_{v}=$ degree of vertex $v$

Definition 1 (see [17]). For a simple connected graph $G$, the $M$-polynomial is defined as follows:

$$
M(G ; x, y)=\sum_{\delta \leq i \leq j \leq \Delta} m_{i j}(G) x^{i} y^{j}
$$

where $\Delta=\operatorname{Min}\left\{d_{v}: v \in V(G)\right\}, \quad \Delta=\operatorname{Max}\left\{d_{v}: v \in V(G)\right\}$, and $m_{i j}(G)$ is the edge $v u \in E(G)$ such that $\left\{d_{v}, d_{u}\right\}=\{i, j\}$.

In 1947, the first topological index was introduced by Wiener, when he was studying the boiling point of alkanes [23]. Subsequently, Wiener established the framework of the topological index $[24,25]$.

After Wiener, in 1975, Milan Randić introduced the Randić index $R_{-1 / 2}(G)$ in [26], which is defined as

$$
R_{-1 / 2}(G)=\sum_{u v \in E(G)} \frac{1}{\sqrt{d_{u} d_{v}}}
$$


and is one of the oldest degree-based topological index. In the year 1998, researchers in [27, 28] introduced the generalized version of the Randić index. The generalized Randić index gets a great attention from a mathematician [29]. We refer to [30] for mathematical properties of this index, and for detailed survey, we refer to [31]. The general Randić index is defined as

$$
R_{\alpha}(G)=\sum_{u v \in E(G)} \frac{1}{\left(d_{u} d_{v}\right)^{\alpha}}
$$

and the generalized inverse Randić index is defined as

$$
R R_{\alpha}(G)=\sum_{u v \in E(G)}\left(d_{u} d_{v}\right)^{\alpha}
$$

If we take $\alpha=-1 / 2$ in the generalized Randić index, we get $R_{-1 / 2}(G)$. The Randić index is likewise the most mainstream regularly connected and most concentrated among all other topological indices. Numerous papers and books, for example, [32-34], are written on this topological index. Randić himself wrote two surveys on his Randić index [35, 36], and there are three more surveys [37-39].

The first and second Zagreb indices are defined by Gutman et al. as

$$
\begin{aligned}
& M_{1}(G)=\sum_{u v \in E(G)}\left(d_{u}+d_{v}\right), \\
& M_{2}(G)=\sum_{u v \in E(G)}\left(d_{u} \times d_{v}\right) .
\end{aligned}
$$

For insight about these indices, we refer [40-44] to the readers. Both the first Zagreb index and the second Zagreb index give more weights to the internal vertices and edges and less weights to external vertices and edges which contradict instinctive thinking. Consequently, they were modified in [45] as the second modified Zagreb index:

$$
{ }^{m} M_{2}(G)=\sum_{u v \in E(G)} \frac{1}{d(u) d(v)} .
$$

The symmetric division index (SDD) is one of the 148 discrete Adriatic indices and is a decent indicator of the aggregate surface area for polychlorobiphenyls [46]. The symmetric division index of a graph $G$ is defined as

$$
\operatorname{SDD}(G)=\sum_{u v \in E(G)}\left\{\frac{\min \left(d_{u}, d_{v}\right)}{\max \left(d_{u}, d_{v}\right)}+\frac{\max \left(d_{u}, d_{v}\right)}{\min \left(d_{u}, d_{v}\right)}\right\} .
$$

Harmonic index is defined as

$$
H(G)=\sum_{v u \in E(G)} \frac{2}{d_{u}+d_{v}} .
$$

As far as we know, this index firstly appeared in [47] and studied in [48].
TABLE 1

\begin{tabular}{lc}
\hline Topological index & Derivation from $M(G ; x, y)$ \\
\hline$M_{1}$ & $\left(D_{x}+D_{y}\right)(M(G ; x, y))_{x=y=1}$ \\
$M_{2}$ & $\left(D_{x} D_{y}\right)(M(G ; x, y))_{x=y=1}$ \\
${ }^{m} M_{2}$ & $\left(S_{x} S_{y}\right)(M(G ; x, y))_{x=y=1}$ \\
$R_{\alpha}$ & $\left(D_{x}^{\alpha} D_{y}^{\alpha}\right)(M(G ; x, y))_{x=y=1}$ \\
$R R_{\alpha}$ & $\left(S_{x}^{\alpha} S_{y}^{\alpha}\right)(M(G ; x, y))_{x=y=1}$ \\
SSD & $\left(D_{x} S_{y}+S_{x} D_{y}\right)(M(G ; x, y))_{x=y=1}$ \\
$H$ & $2 S_{x} J(M(G ; x, y))_{x=1}$ \\
$I$ & $S_{x} J D_{x} D_{y}(M(G ; x, y))_{x=1}$ \\
$A$ & $S_{x}^{3} Q_{-2} J D_{x}{ }^{3} D_{y}^{3}(M(G ; x, y))_{x=1}$ \\
\hline$D_{x}=x(\partial(f(x, y)) / \partial x), D_{y}=y(\partial(f(x, y)) / \partial y), S_{x}=\int_{0}^{x}(f(t, y) / t) d t$, \\
$S_{y}=\int_{0}^{y}(f(x, t) / t) d t, J(f(x, y))=f(x, x)$, and $Q_{\alpha}(f(x, y))=x^{\alpha} f(x, y)$.
\end{tabular}

The inverse sum index is the descriptor that was chosen in [49] as a huge indicator of the aggregate surface area of octane isomers and for which the extremal graphs acquired have an especially straightforward and rich structure. It is denoted by $I$ and is defined as

$$
I(G)=\sum_{v u \in E(G)} \frac{d_{u} d_{v}}{d_{u}+d_{v}}
$$

In [50], Furtula et al. defined the augmented Zagreb index as

$$
A(G)=\sum_{v u \in E(G)}\left\{\frac{d_{u} d_{v}}{d_{u}+d_{v}-2}\right\}^{3}
$$

This graph invariant has wound up being a critical judicious index in the examination of the heat formation in heptanes and octanes (see [50]), whose forecast control is better than the atomic bond connectivity (ABC) index [51-53]. The relationship between $M$-polynomial and indices is presented in Table 1 [17].

\section{Main Results}

In this section, we give our computational results.

3.1. M-Polynomial and Degree-Based Topological Indices of $\mathrm{SC}_{5} \mathrm{C}_{7}[p, q]$

Theorem 1. The M-polynomial of $\mathrm{SC}_{5} \mathrm{C}_{7}[p, q]$ nanotube is

$$
M\left(\mathrm{SC}_{5} \mathrm{C}_{7}[p, q], x, y\right)=p x^{2} y^{2}+[12 p q-9 p] x^{3} y^{3}+6 p x^{2} y^{3}
$$

Proof 1. Then, the vertex set of $\mathrm{SC}_{5} \mathrm{C}_{7}[p, q]$ has the following two partitions with respect to degree: 


$$
\begin{aligned}
& V_{\{2\}}\left(\mathrm{SC}_{5} \mathrm{C}_{7}[p, q]\right)=\left\{\left.v \in V\left(\mathrm{SC}_{5} \mathrm{C}_{7}[p, q]\right)\right|_{d_{v}=2}\right\}, \\
& V_{\{3\}}\left(\mathrm{SC}_{5} \mathrm{C}_{7}[p, q]\right)=\left\{\left.v \in V\left(\mathrm{SC}_{5} \mathrm{C}_{7}[p, q]\right)\right|_{d_{v}=3}\right\} .
\end{aligned}
$$

The edge set of $\mathrm{SC}_{5} \mathrm{C}_{7}[p, q]$ has three partitions based on the degree of end vertices:

$$
\begin{aligned}
& E_{\{2,2\}}\left(\mathrm{SC}_{5} \mathrm{C}_{7}[p, q]\right)=\left\{e=\left.u v \in E\left(\mathrm{SC}_{5} \mathrm{C}_{7}[p, q]\right)\right|_{d_{u}=2, d_{v}=2}\right\}, \\
& E_{\{3,3\}}\left(\mathrm{SC}_{5} \mathrm{C}_{7}[p, q]\right)=\left\{e=\left.u v \in E\left(\mathrm{SC}_{5} \mathrm{C}_{7}[m, n]\right)\right|_{d_{u}=3, d_{v}=3}\right\}, \\
& E_{\{2,3\}}\left(\mathrm{SC}_{5} \mathrm{C}_{7}[p, q]\right)=\left\{e=\left.u v \in E\left(\mathrm{SC}_{5} \mathrm{C}_{7}[p, q]\right)\right|_{d_{u}=2, d_{v}=3}\right\},
\end{aligned}
$$

such that

$$
\begin{aligned}
& \left|E_{\{2,2\}}\left(\mathrm{SC}_{5} \mathrm{C}_{7}[p, q]\right)\right|=p, \\
& \left|E_{\{3,3\}}\left(\mathrm{SC}_{5} \mathrm{C}_{7}[p, q]\right)\right|=12 p q-9 p, \\
& \left|E_{\{2,3\}}\left(\mathrm{SC}_{5} \mathrm{C}_{7}[p, q]\right)\right|=6 p .
\end{aligned}
$$

Now,

$$
\begin{aligned}
M\left(\mathrm{SC}_{5} \mathrm{C}_{7}[p, q], x, y\right)= & \sum_{i \leq j} m_{i j}\left(\mathrm{SC}_{5} \mathrm{C}_{7}[p, q]\right) x^{i} y^{j} \\
= & \sum_{2 \leq 2} m_{22}\left(\mathrm{SC}_{5} \mathrm{C}_{7}[p, q]\right) x^{2} y^{2} \\
& +\sum_{3 \leq 3} m 33\left(\mathrm{SC}_{5} \mathrm{C}_{7}[p, q]\right) x^{3} y^{3} \\
& +\sum_{2 \leq 3} m_{23}\left(\mathrm{SC}_{2} \mathrm{C}_{3}[p, q]\right) x^{2} y^{3} \\
= & \sum_{u v \in E_{\{2,2\}}\left(\mathrm{SC}_{5} \mathrm{C}_{7}[p, q]\right)} m_{22}\left(\mathrm{SC}_{5} \mathrm{C}_{7}[p, q]\right) x^{2} y^{2} \\
& +\sum_{u v \in E_{\{3,3\}}\left(\mathrm{SC}_{5} \mathrm{C}_{7}[p, q]\right)} m_{33}\left(\mathrm{SC}_{5} \mathrm{C}_{7}[p, q]\right) x^{3} y^{3} \\
& +\sum_{u v \in E_{\{2,3\}}\left(\mathrm{SC}_{5} \mathrm{C}_{7}[p, q]\right)} m_{23}\left(\mathrm{SC}_{5} \mathrm{C}_{7}[p, q]\right) x^{2} \\
= & \left|E_{\{2,2\}}\left(\mathrm{SC}_{5} \mathrm{C}_{7}[p, q]\right)\right| x^{2} y^{2} \\
& +\left|E_{\{3,3\}}\left(\mathrm{SC}_{5} \mathrm{C}_{7}[p, q]\right)\right| x^{3} y^{3} \\
& +\left|E_{\{2,3\}}\left(\mathrm{SC}_{5} \mathrm{C}_{7}[p, q]\right)\right| x^{2} y^{3} \\
= & p x^{2} y^{2}+[12 p q-9 p] x^{3} y^{3}+6 p x^{2} y^{3} .
\end{aligned}
$$

Figure 3 shows the behavior of $M$-polynomial of $\mathrm{SC}_{5} \mathrm{C}_{7}[p, q]$ onto the involved parameters $x, y$.

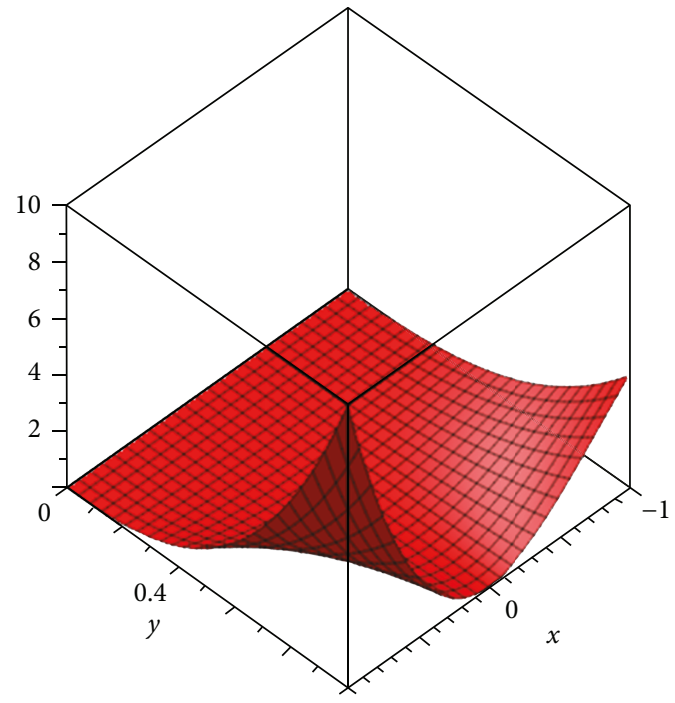

Figure 3: Plot of $M$-polynomial of $\mathrm{SC}_{5} \mathrm{C}_{7}[1,1]$.

Proposition 2. For the nanotube $\mathrm{SC}_{5} \mathrm{C}_{7}[p, q]$, we have

$$
\begin{aligned}
& M_{1}\left(\mathrm{SC}_{5} \mathrm{C}_{7}[p, q]\right)=72 p q-20 p \text {, } \\
& M_{2}\left(\mathrm{SC}_{5} \mathrm{C}_{7}[p, q]\right)=108 p q-41 p \text {, } \\
& { }^{m} M_{2}\left(\mathrm{SC}_{5} \mathrm{C}_{7}[p, q]\right)=\frac{4}{3} p q+\frac{1}{4} p \text {, } \\
& R_{\alpha}\left(\mathrm{SC}_{5} \mathrm{C}_{7}[p, q]\right)=2^{2 \alpha} p+(12 p q-9 p) 3^{2 \alpha}-3^{\alpha} 2^{\alpha} 6 p \text {, } \\
& R R_{\alpha}\left(\mathrm{SC}_{5} \mathrm{C}_{7}[p, q]\right)=\frac{p}{2^{2 \alpha}}+\frac{12}{3^{2 \alpha}} p q-\frac{9}{3^{2 \alpha}} p+\frac{6}{3^{\alpha} \cdot 2^{\alpha}} p \text {, } \\
& \mathrm{SSD}\left(\mathrm{SC}_{5} \mathrm{C}_{7}[p, q]\right)=24 p q-5 p \text {, } \\
& H\left(\mathrm{SC}_{5} \mathrm{C}_{7}[p, q]\right)=4 p q-\frac{1}{10} p, \\
& I\left(\mathrm{SC}_{5} \mathrm{C}_{7}[p, q]\right)=18 p q-\frac{53}{10} p \text {, } \\
& A\left(\mathrm{SC}_{5} \mathrm{C}_{7}[p, q]\right)=\frac{47}{8} p+\frac{27}{2} p q \text {. } \\
& M\left(\mathrm{SC}_{5} \mathrm{C}_{7}[p, q] ; x, y\right)=f(x, y)=p x^{2} y^{2}+6 p x^{2} y^{3} \\
& +(12 p q-9 p) x^{3} y^{3} \text {. }
\end{aligned}
$$

Then,

$$
\begin{aligned}
D_{y} f(x, y) & =2 p x^{2} y^{2}+18 p x^{2} y^{3}+3(12 p q-9 p) x^{3} y^{3}, \\
D_{x} f(x, y) & =2 p x^{2} y^{2}+12 p x^{2} y^{3}+3(12 p q-9 p) x^{3} y^{3}, \\
D_{y} D_{x} f(x, y) & =4 p x^{2} y^{2}+36 p x^{2} y^{3}+9(12 p q-9 p) x^{2} y^{3}, \\
S_{x} S_{y}(f(x, y) & =\frac{1}{4} p x^{2} y^{2}+p x^{2} y^{3}+\frac{1}{9}(12 p q-9 p) x^{3} y^{3}, \\
D_{x}^{a} D_{y}^{a}(f(x, y) & =2^{2 \alpha} p x^{2} y^{2}+2^{\alpha} 3^{\alpha} 6 p x^{2} y^{3}+3^{2 \alpha}(12 p q-9 p) x^{3} y^{3},
\end{aligned}
$$




$$
\begin{aligned}
& S_{x}^{a} S_{y}^{a}(f(x, y))=\frac{1}{2^{2 \alpha}} p x^{2} y^{2}+\frac{6}{2^{\alpha} 3^{\alpha}} p x^{2} y^{3}+\frac{1}{3^{2 \alpha}}(12 p q-9 p) x^{3} y^{3} \\
& S_{y} D_{x}(f(x, y))=p x^{2} y^{2}+\frac{12}{3} p x^{2} y^{3}+(12 p q-9 p) x^{3} y^{3} \\
& S_{x} D_{y}(f(x, y))=p x^{2} y^{2}+9 p x^{2} y^{3}+(12 p q-9 p) x^{3} y^{3} \\
& 2 S_{x} J f(x, y)=2\left[p x^{6}+\frac{6}{5} p x^{5}+(12 p q-9 p) x^{6}\right] \\
& S_{x}^{3} Q_{-2} J D_{x}^{3} D_{y}^{3} f(x, y)=\frac{47}{8} p+\frac{27}{2} p q .
\end{aligned}
$$

Now from Table 1,

(1) First Zagreb index

$$
\begin{aligned}
M_{1}(G) & =\left.\left(D_{x}+D_{y}\right) f(x, y)\right|_{x=y=1} \\
& =14 p+6(12 p q-9 p)+30 p
\end{aligned}
$$

(2) Second Zagreb index

$$
\begin{aligned}
M_{2}(G) & =\left.D_{y} D_{x}(f(x, y))\right|_{x=y=1} \\
& =4 p+9(12 p q-9 p)+36 p
\end{aligned}
$$

(3) Modified second Zagreb index

$$
\begin{aligned}
{ }^{m} M_{2}(G) & =\left.S_{x} S_{y}(f(x, y))\right|_{x=y=1} \\
& =\frac{1}{4} p+\frac{1}{9}(12 p q-9 p)+p
\end{aligned}
$$

(4) Generalized Randić index

$$
\begin{aligned}
R_{\alpha}(G) & =\left.D_{x}^{\alpha} D_{y}^{\alpha}(f(x, y))\right|_{x=y=1} \\
& =2^{2 \alpha} p+3^{2 \alpha}(12 p q-9 p)+2^{\alpha} \cdot 3^{\alpha}(6 p)
\end{aligned}
$$

(5) Inverse Randić index

$$
\begin{aligned}
R R_{\alpha}(G) & =\left.S_{x}^{\alpha} S_{y}^{\alpha}(f(x, y))\right|_{x=y=1} \\
& =\frac{1}{2^{2 \alpha}} p+\frac{2}{3^{2 \alpha}}(12 p q-9 p)+\frac{1}{2^{\alpha} \cdot 3^{\alpha}} 6 p
\end{aligned}
$$

(6) Symmetric division index

$$
\begin{aligned}
\operatorname{SSD}(G) & =\left.\left(S_{y} D_{x}+S_{x} D_{y}\right)(f(x, y))\right|_{x=y=1} \\
& =2 p+2(24 p q-9 p)+4 p+9 p
\end{aligned}
$$

(7) Harmonic index

$$
H(G)=\left.2 S_{x} J(f(x, y))\right|_{x=1}=\frac{1}{4} p+\frac{1}{6}(12 p q-9 p)+\frac{6}{5} p
$$

(8) Inverse sum index

$$
I(G)=S_{x} J D_{x} D_{y}(f(x, y))_{x=1}=p+\frac{3}{2}(12 p q-9 p)+\frac{36}{5} p
$$

(9) Augmented Zagreb index

$$
A(G)=S_{x}{ }^{3} Q_{-2} J D_{x}{ }^{3} D_{y}{ }^{3} f(x, y)=\frac{47}{8} p+\frac{27}{2} p q
$$

3.2. The M-Polynomial and Degree-Based Topological Indices of $\operatorname{NPHX}[p, q]$

Theorem 3. For the H-naphtalenic nanotubes $\operatorname{NPHX}[p, q]$, we have

$$
M(\operatorname{NPHX}[m, n], x, y)=(15 m n-10 m) x^{3} y^{3}+8 m x^{2} y^{3}
$$

Proof 3. The edge set of NPHX $[p, q]$ has the following two partitions with respect to degree of the end vertices:

$$
\begin{aligned}
& E_{\{3,3\}}=\left\{e=\left.u v \in E(\operatorname{NPHX}[p, q])\right|_{d_{u}=3, d_{v}=3}\right\}, \\
& E_{\{2,3\}}=\left\{e=\left.u v \in E(\operatorname{NPHX}[p, q])\right|_{d_{u}=2, d_{v}=3}\right\},
\end{aligned}
$$

such that

$$
\begin{aligned}
& \left|E_{\{3,3\}}\right|=15 p q-10 p, \\
& \left|E_{\{2,3\}}\right|=8 p
\end{aligned}
$$

Now,

$$
\begin{aligned}
M(\operatorname{NPHX}[p, q], x, y)= & \sum_{i \leq j} m_{i j}\left(\operatorname{NPHX}[p, q] x^{i} y^{j}\right) \\
= & \sum_{3 \leq 3} m_{33}(\operatorname{NPHX}[p, q]) x^{3} y^{3} \\
& +\sum_{2 \leq 3} m_{23}(\operatorname{NPHX}[p, q]) x^{2} y^{3} \\
= & \sum_{u v \in E_{\{3,3\}}} m_{33}(\operatorname{NPHX}[p, q]) x^{3} y^{3} \\
& +\sum_{u v \in E_{\{2,3\}}} m_{23}(\operatorname{NPHX}[p, q]) x^{2} y^{3} \\
= & \left|E_{\{3,3\}}\right| x^{3} y^{3}+\left|E_{\{2,3\}}\right| x^{2} y^{3} \\
= & {[15 p q-10 p] x^{3} y^{3}+8 p x^{2} y^{3} . }
\end{aligned}
$$




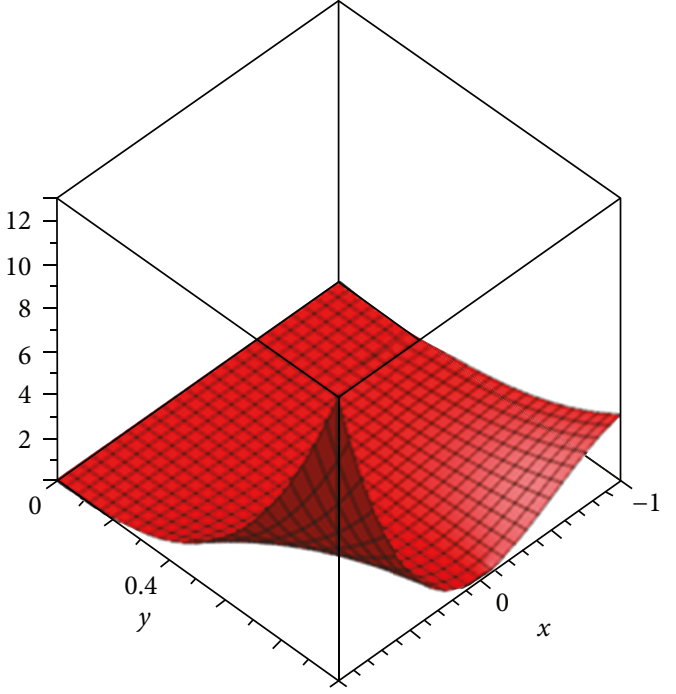

FIgURe 4: Plot of $M$-polynomial of NPHX[1,1].

Figure 4 shows the behavior of $M$-polynomial of NPHX[ $p, q]$, onto the involved parameters $x, y$.

Proposition 4. For the H-naphtalenic nanotubes $\operatorname{NPHX}[p, q]$, we have

$$
\begin{aligned}
M_{1}(\mathrm{NPHX}[p, q]) & =90 p q+20 p, \\
M_{2}(\mathrm{NPHX}[p, q]) & =135 p q-42 p, \\
m_{2}(\operatorname{NPHX}[p, q]) & =\frac{5}{3} p q+\frac{2}{9} p, \\
R_{\alpha}(\operatorname{NPHX}[p, q]) & =3^{\alpha} 3^{\alpha} 15 p q-10 p 3^{2 \alpha}+8 p 2^{\alpha} 3^{\alpha}, \\
R_{\alpha}(\operatorname{NPHX}[p, q]) & =\frac{1}{3^{2 \alpha}}(15 p q-10 p)+\frac{8}{2^{\alpha} \cdot 3^{\alpha}} p, \\
\operatorname{SSD}(\operatorname{NPHX}[p, q]) & =-\frac{8}{3} p+30 p q, \\
H(\operatorname{NPHX}[p, q]) & =-\frac{2}{5} p+5 p q, \\
I(\operatorname{NPHX}[p, q]) & =\frac{45}{2} p q-\frac{27}{5} p, \\
A(\operatorname{NPHX}[p, q]) & =\frac{10935}{64} p q-\frac{1597}{32} p .
\end{aligned}
$$

Proof 4. Let

$$
M(G ; x, y)=f(x, y)=8 p x^{2} y^{3}+(15 p q-8 p) x^{2} y^{3}
$$

Then,

$$
\begin{aligned}
D_{x} f(x, y) & =16 p x^{2} y^{3}+3(15 p q-10 p) x^{3} y^{3}, \\
D_{y} f(x, y) & =24 p x^{2} y^{3}+3(15 p q-10 p) x^{3} y^{3}, \\
S_{x} S_{y}(f(x, y)) & =\frac{8}{6} p x^{2} y^{3}+\frac{1}{9}(15 p q-10 p) x^{3} y^{3},
\end{aligned}
$$

$$
\begin{aligned}
D_{x}^{a} D_{y}^{a}(f(x, y)) & =2^{\alpha} 3^{\alpha} 8 p x^{2} y^{3}+3^{2 \alpha}(15 p q-10 p) x^{3} y^{3}, \\
S_{x}^{a} S_{y}^{a}(f(x, y)) & =\frac{8}{2^{\alpha} 3^{\alpha}} p x^{2} y^{3}+\frac{1}{3^{2 \alpha}}(15 p q-10 p) x^{3} y^{3}, \\
S_{y} D_{x}(f(x, y)) & =\frac{16}{3} p x^{2} y^{3}+(15 p q-10 p) x^{3} y^{3}, \\
2 S_{x} J f(x, y) & =2\left[\frac{8}{5} p x^{5}+\frac{1}{6}(15 p q-10 p) x^{6}\right], \\
S_{x} J D_{x} D_{y} f(x, y) & =\frac{48}{5} p x^{5}+\frac{9}{6}(15 p q-10 p) x^{3} y^{3}, \\
S_{x}^{3} Q_{-2} J D_{x}^{3} D_{y}^{3} f(x, y) & =\frac{1728}{27} p x^{3}-\frac{7290}{64}(15 p q-10 p) x^{4} .
\end{aligned}
$$

(1) First Zagreb index

$$
\begin{aligned}
M_{1}(\operatorname{NPHX}[p, q]) & =\left.\left(D_{x}+D_{y}\right) f(x, y)\right|_{x=y=1} \\
& =6(15 p q-10 p)+24 p
\end{aligned}
$$

(2) Second Zagreb index

$$
\begin{aligned}
M_{2}(\operatorname{NPHX}[p, q]) & =\left.D_{y} D_{x}(f(x, y))\right|_{x=y=1} \\
& =9(15 p q-10 p)+48 p
\end{aligned}
$$

(3) Modified second Zagreb index

$$
\begin{aligned}
{ }^{m} M_{2}(\operatorname{NPHX}[p, q]) & =\left.S_{x} S_{y}(f(x, y))\right|_{x=y=1} \\
& =\frac{1}{9} p+\frac{1}{6}(15 p q-10 p)
\end{aligned}
$$

(4) Generalized Randić index

$$
\begin{aligned}
R_{\alpha}(\operatorname{NPHX}[p, q]) & =\left.D_{x}^{\alpha} D_{y}^{\alpha}(f(x, y))\right|_{x=y=1} \\
& =3^{2 \alpha}(15 p q-10 p)+2^{\alpha} \cdot 3^{\alpha}(8 p)
\end{aligned}
$$

(5) Inverse Randić index

$$
\begin{aligned}
R R_{\alpha}(\operatorname{NPHX}[p, q]) & =\left.S_{x}^{\alpha} S_{y}^{\alpha}(f(x, y))\right|_{x=y=1} \\
& =\frac{1}{3^{2 \alpha}}(15 p q-10 p)+\frac{1}{2^{\alpha} \cdot 3^{\alpha}} 8 p
\end{aligned}
$$




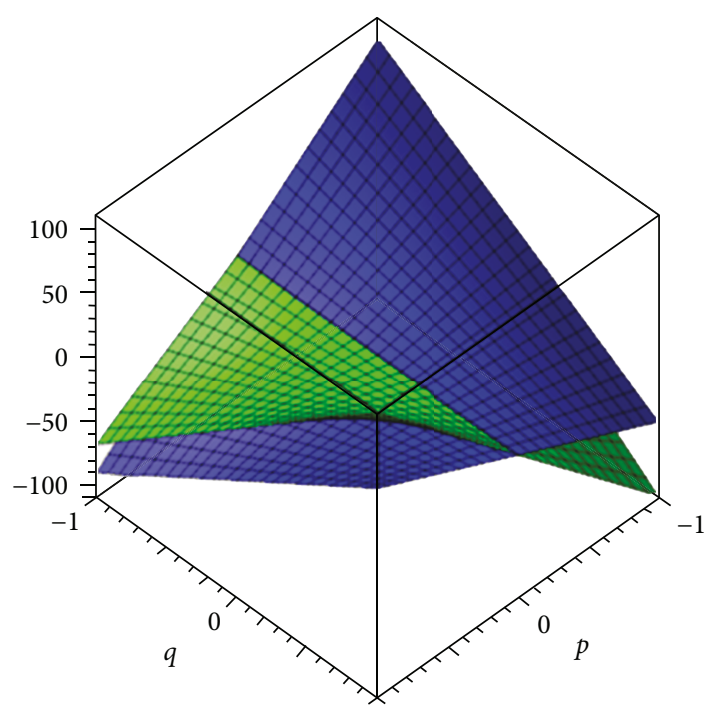

Figure 5: Plots of first Zagreb indices.

(6) Symmetric division index

$$
\begin{aligned}
\operatorname{SSD}(\operatorname{NPHX}[p, q]) & =\left.\left(S_{y} D_{x}+S_{x} D_{y}\right)(f(x, y))\right|_{x=y=1} \\
& =2(15 p q)-\frac{8}{3} p
\end{aligned}
$$

(7) Harmonic index

$$
H(\operatorname{NPHX}[p, q])=\left.2 S_{x} J(f(x, y))\right|_{x=1}
$$

(8) Inverse sum index $=\frac{1}{6}(15 p q-10 p)+\frac{1}{5} 8 p$

$$
\begin{aligned}
I(\operatorname{NPHX}[p, q]) & =S_{x} J D_{x} D_{y}(f(x, y))_{x=1} \\
& =\frac{3}{2}(15 p q-10 p)+\frac{48}{5} p
\end{aligned}
$$

(9) Augmented Zagreb index

$$
\begin{aligned}
A(\operatorname{NPHX}[p, q]) & =\left.S_{x}^{3} Q_{-2} J D_{x}^{3} D_{y}^{3}(f(x, y))\right|_{x=1} \\
& =\frac{10935}{64} p q-\frac{1597}{32} p
\end{aligned}
$$

\section{Discussion and Concluding Remarks}

We computed $M$-polynomials of $\mathrm{SC}_{5} \mathrm{C}_{7}[p, q]$ and $\mathrm{NPHX}[p$, $q$ ]. From these $M$-polynomials, we recovered nine degreebased topological indices by applying fundamental rules of calculus. Our calculated results help to understand topology of understudy nanotubes. For instant, the first Zagreb index is used to known about pi-electronic energy. Augmented Zagreb index can be used in the investigation of the heat of formation. Randić index is the most applied and investigated degree-based topological index. Figures 5-13 show the

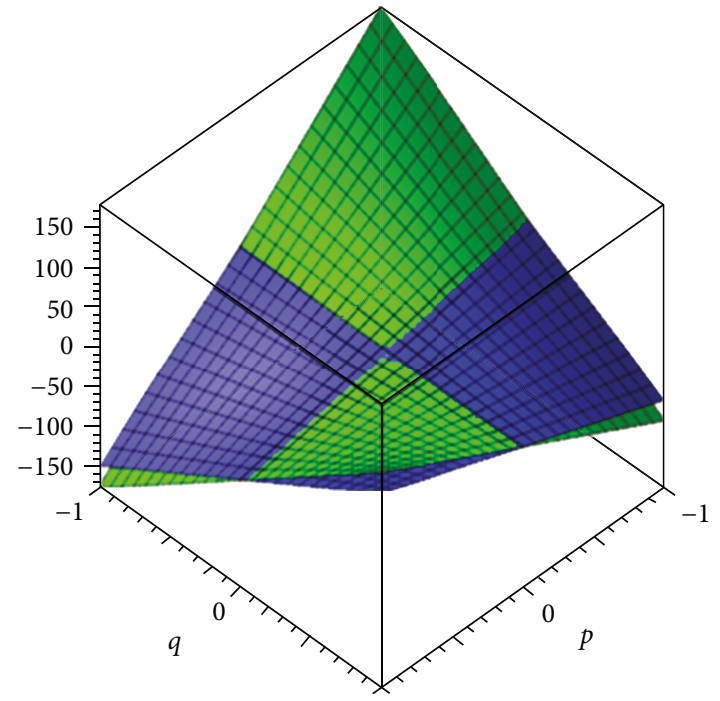

Figure 6: Plots of second Zagreb indices.

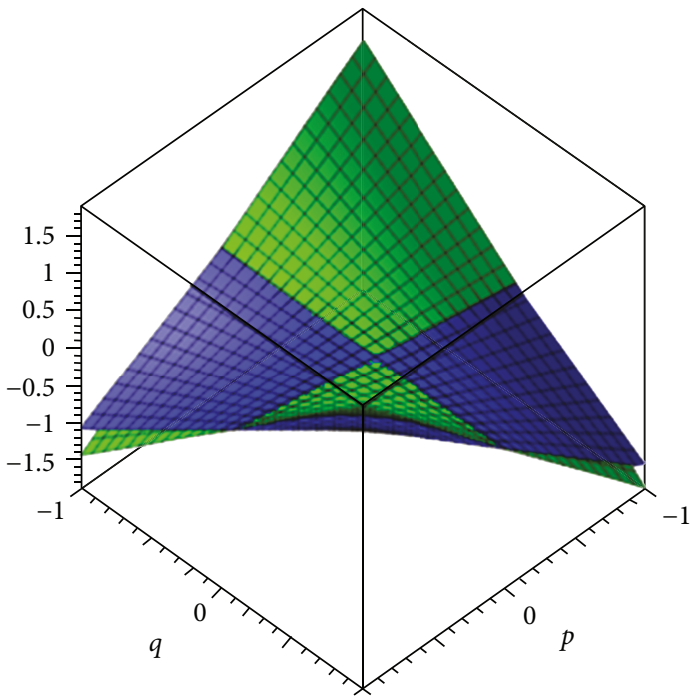

Figure 7: Plots of modified second Zagreb indices.

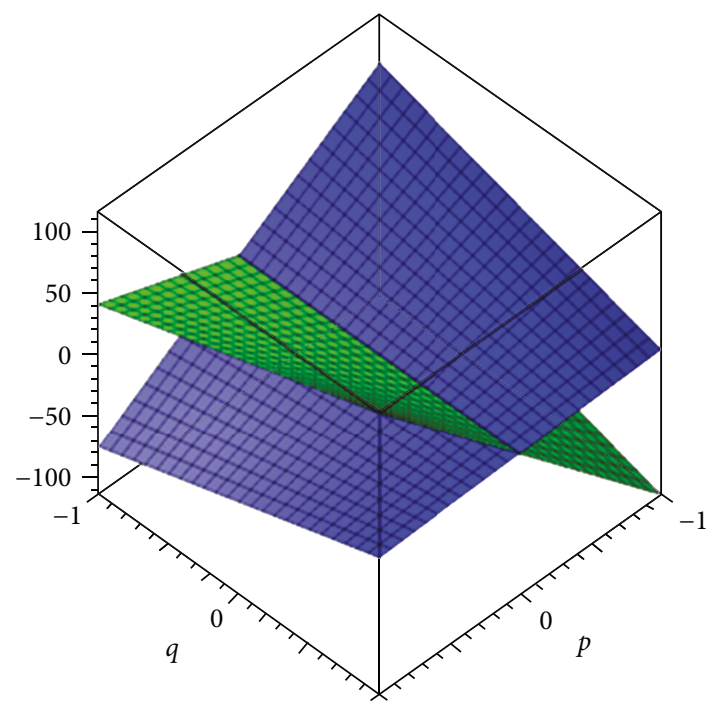

Figure 8: Plots of generalized Randić indices for alpha =1/2. 


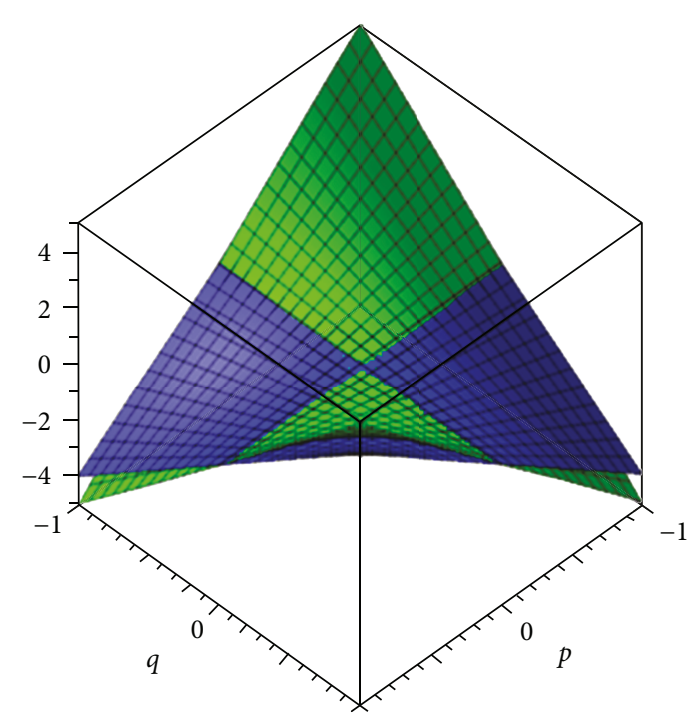

Figure 9: Plots of inverse Randić indices for alpha $=1 / 2$.

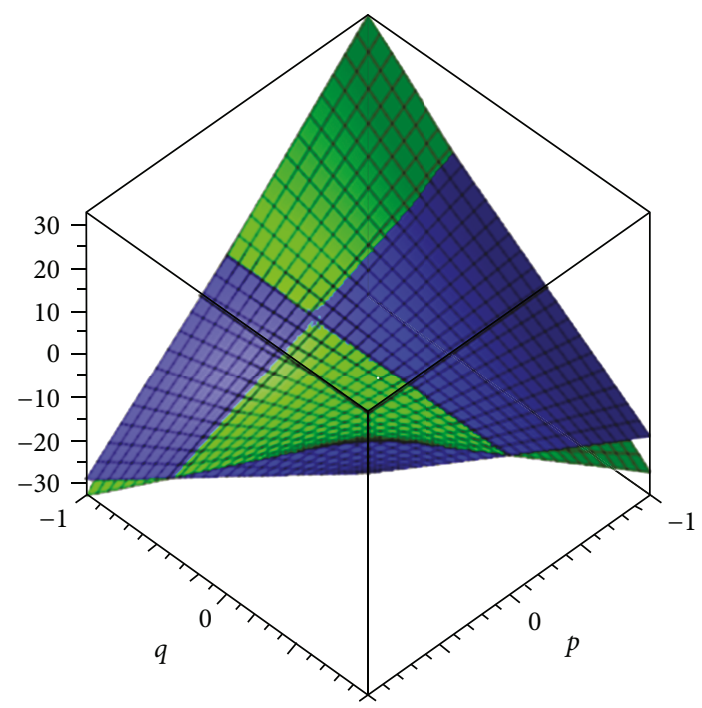

FIgUre 10: Plots of symmetric division indices.

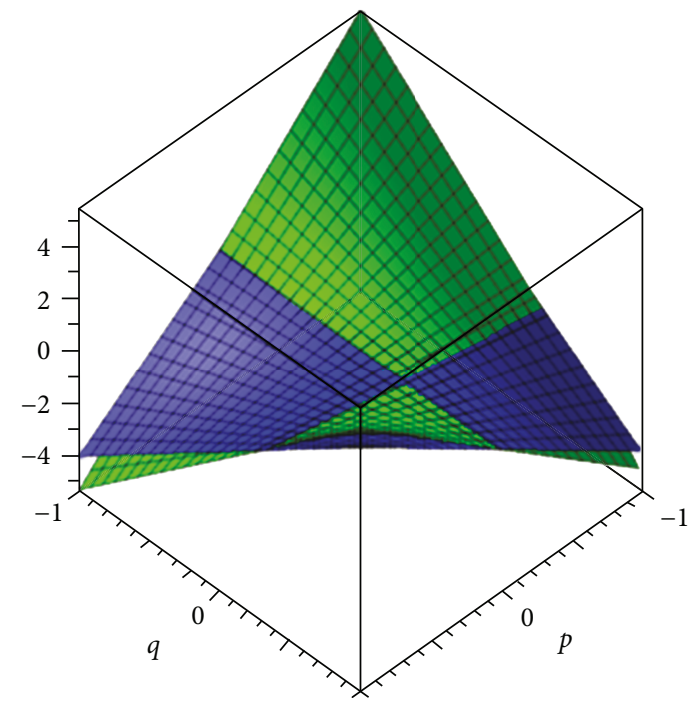

FIGURE 11: Plots of harmonic indices.

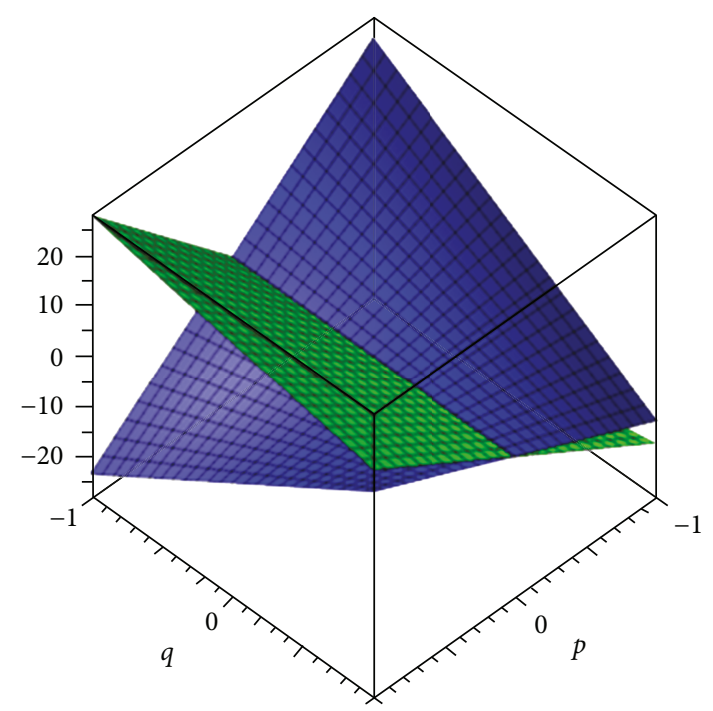

Figure 12: Plots of inverse sum indices.

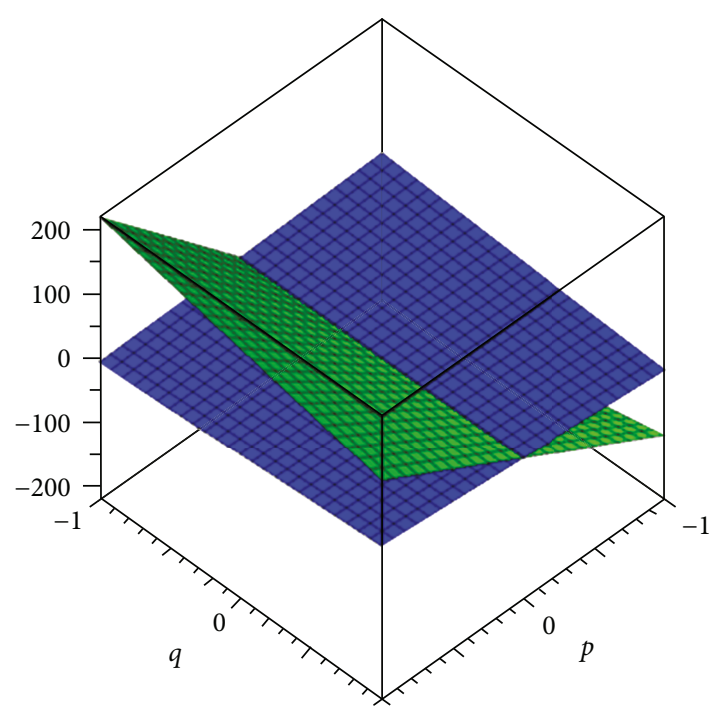

FIGURE 13: Plots of augmented Zagreb indices.

dependence of our results on the involved parameters. Blue color is fixed for $\mathrm{SC}_{5} \mathrm{C}_{7}[p, q]$, and green color is fixed for $\operatorname{NPHX}[p, q]$.

\section{Data Availability}

All data is included within this paper.

\section{Conflicts of Interest}

The authors do not have any competing interests.

\section{Authors' Contributions}

All authors contribute equally in this paper. 


\section{Acknowledgments}

The work was supported in part by funding. This research was funded by the Natural Science Research Key Project from Education Department of Anhui Province (Grant No. KJ2017A492) and youth research special fund project of Anhui Jianzhu University (Grant No. 2011183-8).

\section{References}

[1] H. Deng, X. Li, Q. Peng, X. Wang, J. Chen, and Y. Li, "Monodisperse magnetic single-crystal ferrite microspheres," Angewandte Chemie, vol. 117, no. 18, pp. 2842-2845, 2005.

[2] G. A. Somorjai and Y. G. Borodko, "Research in nanosciencesgreat opportunity for catalysis science," Catalysis Letters, vol. 76 , no. $1 / 2$, pp. $1-5,2001$.

[3] R. J. P. Corriu, A. Mehdi, and C. Reyé, "Molecular chemistry and nanosciences: on the way to interactive materials," Journal of Materials Chemistry, vol. 15, no. 40, pp. 4285-4294, 2005.

[4] R. H. Baughman, A. A. Zakhidov, and W. A. De Heer, "Carbon nanotubes-the route toward applications," Science, vol. 297, no. 5582, pp. 787-792, 2002.

[5] B. S. Harrison and A. Atala, "Carbon nanotube applications for tissue engineering," Biomaterials, vol. 28, no. 2, pp. 344353, 2007.

[6] M. F. L. De Volder, S. H. Tawfick, R. H. Baughman, and A. J. Hart, "Carbon nanotubes: present and future commercial applications," Science, vol. 339, no. 6119, pp. 535-539, 2013.

[7] A. K. T. Lau and D. Hui, "The revolutionary creation of new advanced materials-carbon nanotube composites," Composites Part B: Engineering, vol. 33, no. 4, pp. 263-277, 2002.

[8] H. Hui-Fang, L. Yi-bing, Z. Wei-ya, and T. Dong-sheng, "Electronic properties of carbon nanotubes with a pentagonheptagon pair defect," Chinese Physics, vol. 10, no. 6, pp. 531-536, 2001.

[9] W. Gao, W. Wang, M. K. Jamil, and M. R. Farahani, "Electron energy studying of molecular structures via forgotten topological index computation," Journal of Chemistry, vol. 2016, 7 pages, 2016.

[10] D. B. West, An Introduction to Graph Theory, Prentice-Hall, 1996.

[11] G. Rucker and C. Rucker, "On topological indices, boiling points, and cycloalkanes," Journal of Chemical Information and Computer Sciences, vol. 39, no. 5, pp. 788-802, 1999.

[12] S. Klavžar and I. Gutman, "A comparison of the Schultz molecular topological index with the Wiener index," Journal of Chemical Information and Computer Sciences, vol. 36, no. 5, pp. 1001-1003, 1996.

[13] F. M. Brückler, T. Došlić, A. Graovac, and I. Gutman, "On a class of distance-based molecular structure descriptors," Chemical Physics Letters, vol. 503, no. 4-6, pp. 336-338, 2011.

[14] H. Deng, J. Yang, and F. Xia, "A general modeling of some vertex-degree based topological indices in benzenoid systems and phenylenes," Computers \& Mathematics with Applications, vol. 61, no. 10, pp. 3017-3023, 2011.

[15] H. Zhang and F. Zhang, "The Clar covering polynomial of hexagonal systems I," Discrete Applied Mathematics, vol. 69, no. 12, pp. 147-167, 1996.

[16] I. Gutman, "Some properties of the Wiener polynomials," Graph Theory Notes New York, vol. 125, pp. 13-18, 1993.
[17] E. Deutsch and S. Klavzar, "M-polynomial, and degree-based topological indices," Iranian Journal of Mathematical Chemistry, vol. 6, pp. 93-102, 2015.

[18] M. Munir, W. Nazeer, S. Rafique, and S. M. Kang, "M-polynomial and related topological indices of nanostar dendrimers," Symmetry, vol. 8, no. 9, p. 97, 2016.

[19] M. Munir, W. Nazeer, S. Rafique, A. R. Nizami, and S. M. Kang, "M-polynomial and degree-based topological indices of titania nanotubes," Symmetry, vol. 8, no. 11, p. 117, 2016.

[20] M. Munir, W. Nazeer, S. Rafique, and S. M. Kang, "M-polynomial and degree-based topological indices of polyhex nanotubes," Symmetry, vol. 8, no. 12, p. 149, 2016.

[21] M. R. Farahani, M. K. Jamil, and M. Imran, "Vertex PIv topological index of titania carbon nanotubes $\mathrm{TiO} 2(\mathrm{~m}, \mathrm{n})$," Applied Mathematics and Nonlinear Sciences, vol. 1, no. 1, pp. 175-2845, 2016.

[22] M. Munir, W. Nazeer, S. Shahzadi, and S. M. Kang, "Some invariants of circulant graphs," Symmetry, vol. 8, no. 11, p. 134, 2016.

[23] H. Wiener, "Structural determination of paraffin boiling points," Journal of the American Chemical Society, vol. 69, no. 1, pp. 17-20, 1947.

[24] A. A. Dobrynin, R. Entringer, and I. Gutman, "Wiener index of trees: theory and applications," Acta Applicandae Mathematica, vol. 66, no. 3, pp. 211-249, 2001.

[25] I. Gutman and O. E. Polansky, Mathematical Concepts in Organic Chemistry, Springer-Verlag New York, New York, NY, USA, 1986.

[26] M. Randić, "Characterization of molecular branching," Journal of the American Chemical Society, vol. 97, no. 23, pp. 6609-6615, 1975.

[27] B. Bollobás and P. Erdös, "Graphs of extremal weights," Ars Combinatoria, vol. 50, pp. 225-233, 1998.

[28] D. Amić, D. Beslo, B. Lučić, S. Nikolić, and N. Trinajstić, "The vertex-connectivity index revisited," Journal of Chemical Information and Computer Sciences, vol. 38, no. 5, pp. 819-822, 1998.

[29] Y. Hu, X. Li, Y. Shi, T. Xu, and I. Gutman, "On molecular graphs with smallest and greatest zeroth-order general Randić index," MATCH Communications in Mathematical and in Computer Chemistry, vol. 54, pp. 425-434, 2005.

[30] G. Caporossi, I. Gutman, P. Hansen, and L. Pavlovic, "Graphs with maximum connectivity index," Computational Biology and Chemistry, vol. 27, no. 1, pp. 85-90, 2003.

[31] M. Alaeiyan, M. R. Farahani, and M. K. Jamil, "Computation of the fifth geometric-arithmetic index for polycyclic aromatic hydrocarbons PAHk," Applied Mathematics and Nonlinear Sciences, vol. 1, no. 1, pp. 283-290, 2016.

[32] L. B. Kier and L. H. Hall, Molecular Connectivity in Chemistry and Drug Research, Academic Press, New York, 1976.

[33] L. B. Kier and L. H. Hall, Molecular Connectivity in StructureActivity Analysis, Wiley, New York, 1986.

[34] X. Li and I. Gutman, Mathematical Aspects of Randić-Type Molecular Structure Descriptors, University of Kragujevac, Kragujevac, 2006.

[35] M. Randić, "On history of the Randić index and emerging hostility toward chemical graph theory," MATCH Communications in Mathematical and in Computer Chemistry, vol. 59, pp. 5-124, 2008. 
[36] M. Randić, “The connectivity index 25 years after," Journal of Molecular Graphics \& Modelling, vol. 20, no. 1, pp. 19-35, 2001.

[37] I. Gutman and B. Furtula, Recent Results in the Theory of Randić Index, University of Kragujevac, Kragujevac, 2008.

[38] X. Li and Y. Shi, "A survey on the Randić index," MATCH Communications in Mathematical and in Computer Chemistry, vol. 59, pp. 127-156, 2008.

[39] X. Li, Y. Shi, and L. Wang, Recent Results in the Theory of Randić Index, I. Gutman and B. Furtula, Eds., University of Kragujevac, Kragujevac, 2008.

[40] S. Nikolić, G. Kovačević, A. Miličević, and N. Trinajstić, “The Zagreb indices 30 years after," Croatica Chemica Acta, vol. 76, pp. 113-124, 2003.

[41] I. Gutman and K. C. Das, "The first Zagreb indices 30 years after," MATCH Communications in Mathematical and in Computer Chemistry, vol. 50, pp. 83-92, 2004.

[42] K. Das and I. Gutman, "Some properties of the second Zagreb index," MATCH Communications in Mathematical and in Computer Chemistry, vol. 52, pp. 103-112, 2004.

[43] N. Trinajstić, S. Nikolić, A. Miličević, and I. Gutman, "On Zagreb indices," Kemija u Industriji, vol. 59, pp. 577-589, 2010.

[44] D. Kovačević and A. Graovac, "Valence connectivities versus Randić, Zagreb and modified Zagreb index: a linear algorithm to check discriminative properties of indices in acyclic molecular graphs," Croatica Chemica Acta, vol. 77, pp. 501-508, 2004.

[45] A. Miličević, S. Nikolić, and N. Trinajstić, "On reformulated Zagreb indices," Molecular Diversity, vol. 8, no. 4, pp. 393399, 2004.

[46] C. K. Gupta, V. Lokesha, S. B. Shwetha, and P. S. Ranjini, "On the symmetric division deg index of graph," Southeast Asian Bulletin of Mathematics, vol. 40, no. 1, pp. 59-80, 2016, 22p.

[47] W. Gao and M. R. Farahani, "Degree-based indices computation for special chemical molecular structures using edge dividing method," Applied Mathematics and Nonlinear Sciences, vol. 1, no. 1, pp. 99-122, 2016.

[48] O. Favaron, M. Mahéo, and J. F. Saclé, "Some eigenvalue properties in graphs (conjectures of Graffiti-II)," Discrete Mathematics, vol. 111, no. 1-3, pp. 197-220, 1993.

[49] A. T. Balaban, "Highly discriminating distance based numerical descriptor," Chemical Physics Letters, vol. 89, pp. 399-404, 1982.

[50] B. Furtula, A. Graovac, and D. Vukičević, “Augmented Zagreb index," Journal of Mathematical Chemistry, vol. 48, no. 2, pp. 370-380, 2010.

[51] K. C. Das, “Atom-bond connectivity index of graphs," Discrete Applied Mathematics, vol. 158, no. 11, pp. 1181-1188, 2010.

[52] E. Estrada, L. Torres, L. Rodriguez, and I. Gutman, “An atombond connectivity index: modeling the enthalpy of formation of alkanes," Indian Journal of Chemistry, vol. 37A, pp. 849$855,1998$.

[53] E. Estrada, "Atom-bond connectivity and the energetic of branched alkanes," Chemical Physics Letters, vol. 463, no. 46, pp. 422-425, 2008. 


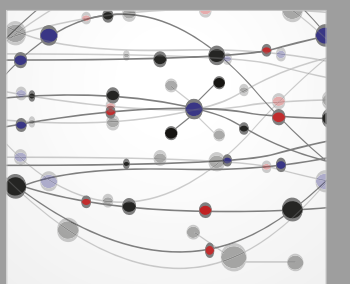

The Scientific World Journal
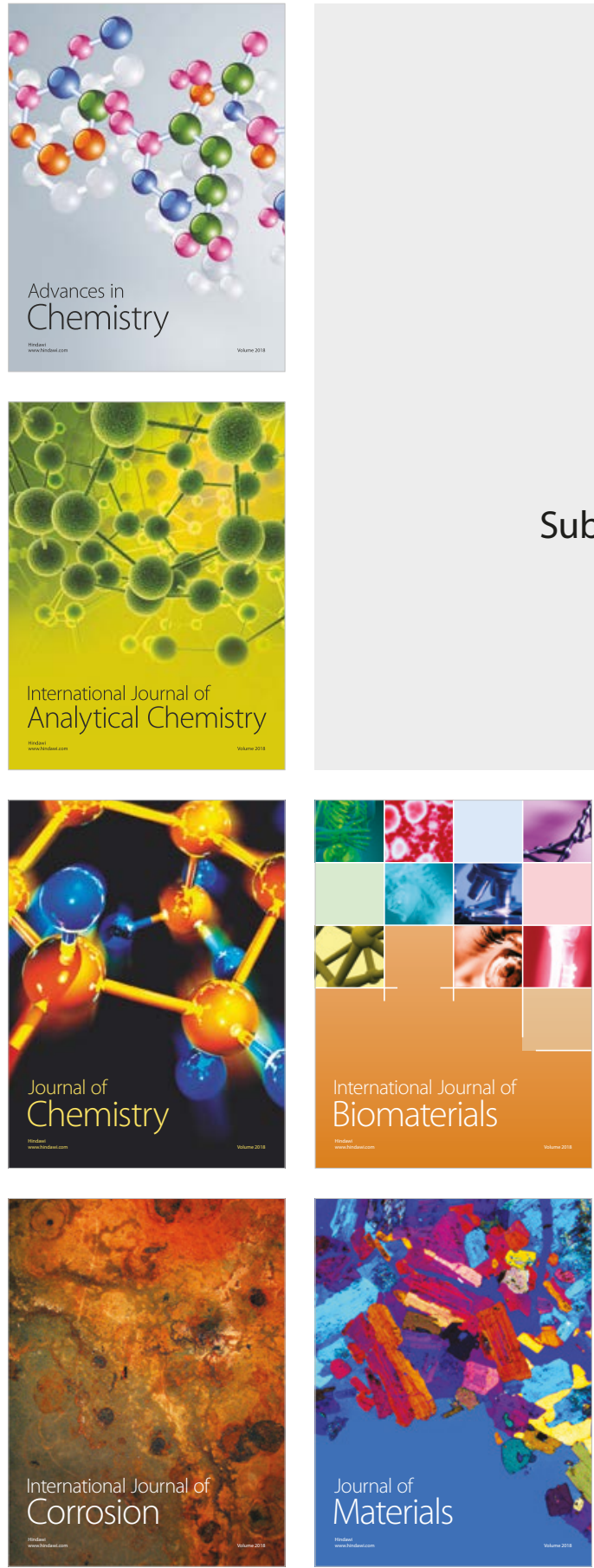

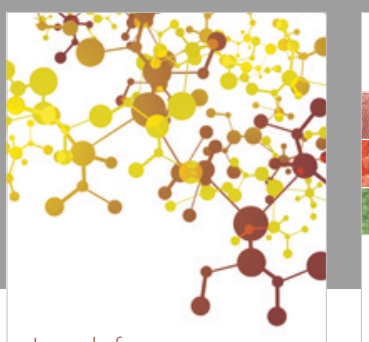

Journal of

Applied Chemistry
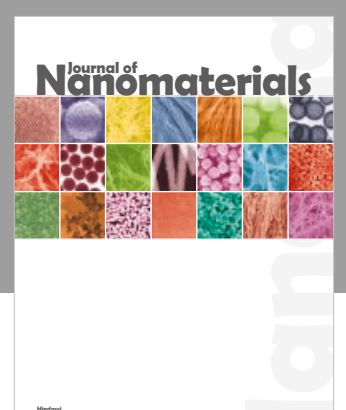

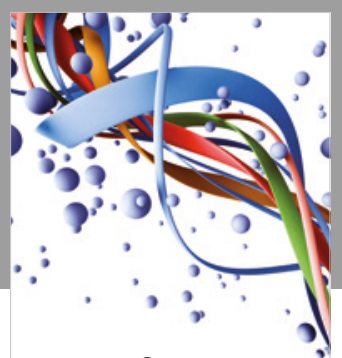

Scientifica

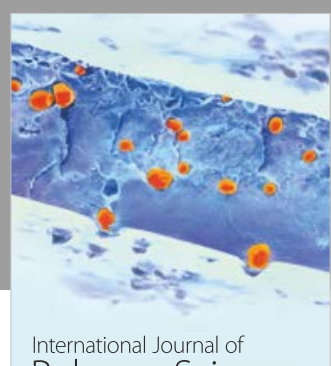

Polymer Science

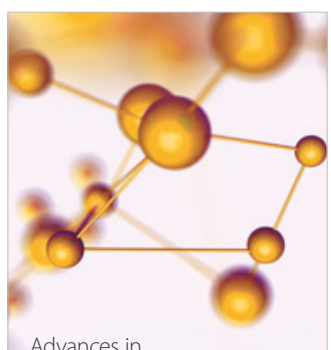

Physical Chemistry
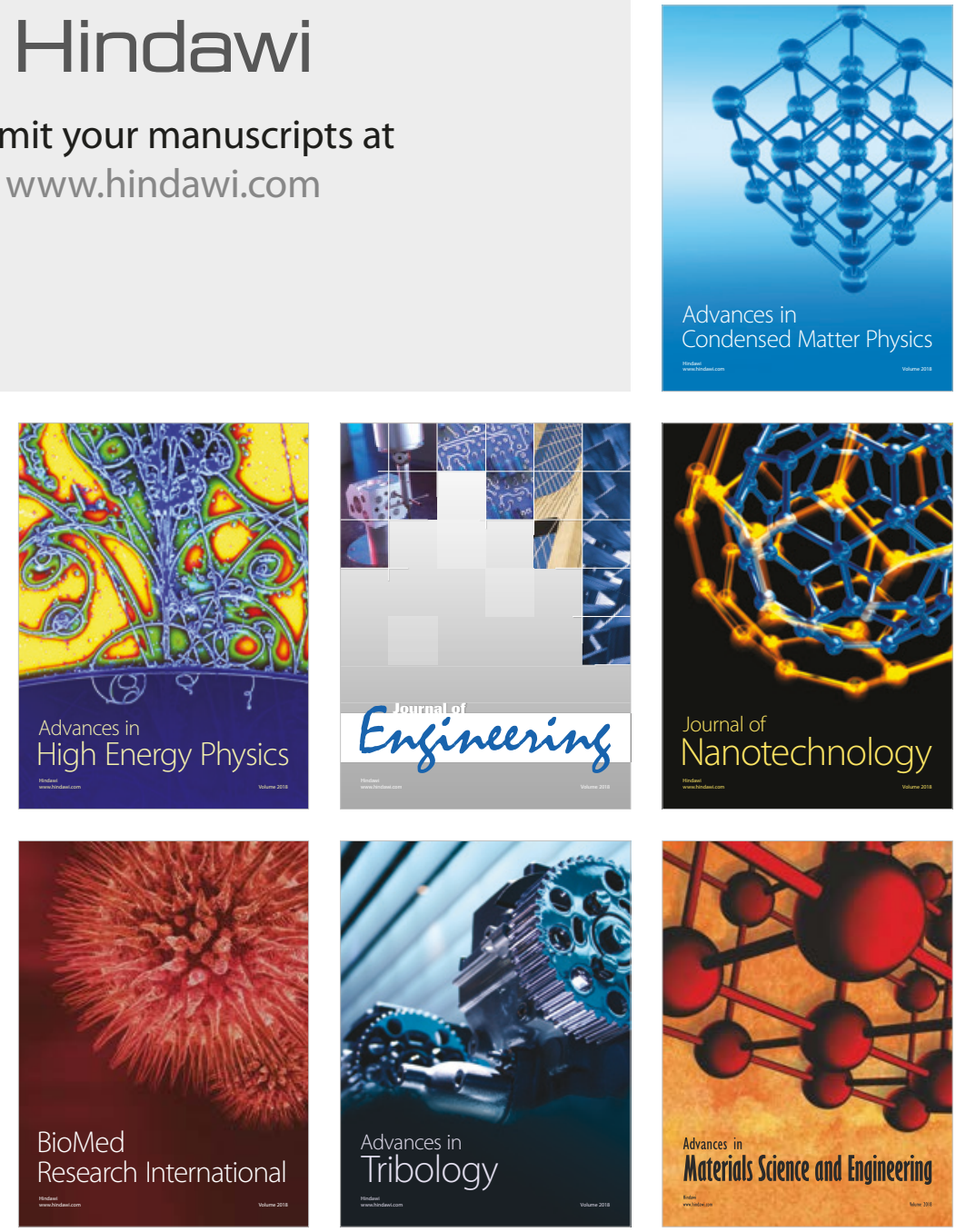